\title{
Universiteit
}

Leiden

The Netherlands

\section{Active mechanical stabilization of the viscoplastic intracellular space of Dictyostelia cells by microtubule-actin crosstalk}

Heinrich, D.M.; Sackmann, E.

\section{Citation}

Heinrich, D. M., \& Sackmann, E. (2006). Active mechanical stabilization of the viscoplastic intracellular space of Dictyostelia cells by microtubule-actin crosstalk. Acta Biomaterialia, 2, 619-631. doi:10.1016/j.actbio.2006.05.014

Version: $\quad$ Not Applicable (or Unknown)

License: $\quad$ Leiden University Non-exclusive license

Downloaded from: https://hdl.handle.net/1887/53026

Note: To cite this publication please use the final published version (if applicable). 


\title{
Active mechanical stabilization of the viscoplastic intracellular space of Dictyostelia cells by microtubule-actin crosstalk
}

\author{
Doris Heinrich ${ }^{a}$, Erich Sackmann ${ }^{b, *}$ \\ ${ }^{a}$ Physik Department, Lehrstuhl fuer Biophysik E22, Technische Universitaet Muenchen, D-85748 Garching, Germany \\ ${ }^{\mathrm{b}}$ Sektion Physik, Lehrstuhl fuer Angewandte Physik, Universitaet Muenchen, Amalienstrasse 54, D-80799 Muenchen, Germany
}

Received 1 January 2006; received in revised form 19 May 2006; accepted 23 May 2006

\begin{abstract}
The micro-viscoelasticity of the intracellular space of Dictyostelium discoideum cells is studied by evaluating the intracellular transport of magnetic force probes and their viscoelastic responses to force pulses of $20-700 \mathrm{pN}$. The role of the actin cortex, the microtubule (MT) aster and their crosstalk is explored by comparing the behaviour of wild-type cells, myosin II null mutants, latrunculin A and benomyl treated cells. The MT coupled beads perform irregular local and long range directed motions which are characterized by measuring their velocity distributions $(P(v))$. The correlated motion of the MT and the centrosome are evaluated by microfluorescence of GFP-labelled MTs. $P(v)$ can be represented by log-normal distributions with long tails and it is determined by random sweeping motions $(v \sim 0.5 \mu \mathrm{m} / \mathrm{s})$ of the MTs (caused by tangential forces on the filament ends coupled to the actin cortex) and by intermittent bead transports parallel to the MTs $\left(v_{\max } \sim 1.5 \mu \mathrm{m} / \mathrm{s}\right)$. The tails are due to spontaneous filament deflections (with speeds up to $10 \mu \mathrm{m} / \mathrm{s}$ ) attributed to pre-stressing of the MT by local cortical tensions, generated by dynactin motors generating plus-end directed forces in the MTs. The viscoelastic responses are strongly non-linear and are mostly directed opposite or perpendicular to the force, showing that the cytoplasm behaves as an active viscoplastic body with time and force dependent drag coefficients. Nano-Newton loads exerted on the soft MT are balanced by traction forces arising at the MT ends coupled to the actin cortex and the centrosome, respectively. The mechanical coupling between the soft microtubules and the viscoelastic actin cortex provides cells with high mechanical stability despite the softness of the cytoplasm.
\end{abstract}

(c) 2006 Acta Materialia Inc. Published by Elsevier Ltd. All rights reserved.

Keywords: Magnetic tweezers; Microrheometry of living cells; Viscoplasticity of cytoplasm; Microtubule-actin crosstalk; Intracellular transport forces

\section{Introduction}

Quantitative studies of micro-mechanical properties of cells are becoming of growing interest for several reasons. First, mechanical material properties play a key role in the control of numerous cellular processes such as cell shape changes [1], cell locomotion [2], adhesion [3] or cell division [4] by mechanical forces. Second, local forces can control the cellular architecture in a subtle way, for instance through the activation of small GTPases of the rho-family [5] which can result in rapid (sub-second) re-

\footnotetext{
* Corresponding author. Tel.: +498928912490.

E-mail address: sackmann@ph.tum.de (E. Sackmann).
}

organizations of the actin network [6], including the formation of stress fibres [7], the activation of micro-muscles or the growth of cellular protrusions, such as philipodia [8] or axons [9]. Finally, measurements of viscoelastic moduli provide a sensitive tool for real time studies of structural re-organizations of the cytoskeleton induced by external forces, cell signalling processes, or biochemical perturbations $[7,10]$. Micromechanical studies can also provide an answer to the long standing and controversial question of whether information can be transmitted from the cell envelope to the nucleus by elastic stresses [11].

Various micromechanical techniques have been established during the last few years to measure local elastic moduli or the stress-strain relationship of whole cells 
including micropipette aspiration techniques [12-14], atomic force microscopy [15], optical tweezers [16,17], or cell poking with glass rods [18]. Based on these techniques, the elastic properties of cell envelopes, whole nucleated cells and erythrocytes have been extensively studied $[1,19]$. The findings of these experiments may be summarized by the statement that nucleated cells can be described in terms of droplets of polymeric fluid which are enclosed by elastic shells exhibiting bending and shear elasticity $[13,14,20]$.

In contrast, the viscoelasticity of cells or cellular substructures has only gained broad interest more recently despite its key role in the evolution of the mechanical control of cellular processes over time. Moreover, studies of the local viscoelastic moduli of the cell envelope or the cytoplasm can yield valuable insights into the structure of the membrane associated actin cortex and structural changes induced by cell stimulating or damaging agents [7,10], deceases [21] or by application of external forces [22]. A variety of colloidal probe microrheometry techniques have been developed over the last few years based on the analysis of Brownian motion $[10,23,24]$ or of the viscoelastic responses evoked by local forces applied through optical traps $[10,23]$ and magnetic tweezers. With magnetic tweezers, viscoelastic responses can be evoked by linear [25] or by torsional $[26,27]$ excitations. The viscoelastic response to force pulses yield relaxation moduli $G(t)$ and relaxation times, while oscillatory force scenarios yield complex viscoelastic impedances. Since the two sets of parameters are related by convolution, the two modes of excitation yield equivalent results [7].

Judged from micromechanical studies, cells can be considered to be built-up of three mechanically distinct bodies [19]: the cell envelope, the cytoplasm and the nuclear complex. Cell envelopes behave as linear viscoelastic bodies, the viscoelastic impedance of which exhibit the typical viscoelastic signatures of actin networks [7]. The cytoplasmic space is a soft colloidal assembly which is mechanically stabilized by the aster-shaped microtubule (MT) network. In mammalian cells its stability is enforced by the network of intermediate filaments while this system is missing in Dictyostelia cells. The nuclear envelope forms again a more rigid viscoelastic body [28].

The present work was motivated by the question of whether, similar to the cell envelope, the intracellular space behaves as a true viscoelastic body. Microrheometric studies of the cytoplasm of mammalian cells by magnetic tweezer microrheometry [25] and force free laser tracking [10] have been interpreted in terms of the models of viscoelastic bodies exhibiting shear moduli of the order of $100 \mathrm{~Pa}$. However, shear field mapping experiments did not provide any evidence for elastic coupling between intracellular compartments over distances of $0.5 \mu \mathrm{m}$ [25]. More recent microrheological studies of Dictyostelia cells immobilized by agar overlays provided evidence that the cytoplasmic space behaves rather as a viscoplastic body [29]. To gain more detailed insight into the nature of the dynamic mechanical properties of the cytoplasmic space, we studied wild-type $D$. discoideum cells, cells with GFP labelled microtubules and mutants lacking the motor protein myosin II under more natural conditions. The quasi-spherical vegetative cells were settled onto glass substrates but not compressed by agar overlays. Three series of experiments were performed. First, we measured the local speeds and mean square displacements of actively transported superparamagnetic beads of $1.42 \mu \mathrm{m}$ diameter over extended periods of time (20-40 min). The beads are engulfed by the cells through phagocytosis. They become coated by the plasma membrane exposing the cytoplasmic surface of the cell membrane and are therefore expected to be processed by the cell similar to intracellular compartments. To study the control of the intracellular transport of the magnetosomes by microtubules, their motion and their conformational changes were visualized by fluorescence microscopy of cells transfected with green fluorescent tubulin (GFP- $\alpha$-tubulin).

In a second set of experiments the viscoelastic response of the beads evoked by force pulses of $20-700 \mathrm{pN}$ were studied. The beads visited some regions repeatedly enabling us to observe the bead trajectory at the same local region within the cell in the absence and presence of external forces and to measure viscosities and active forces inside living cells. To gain some insight into the control of the mechanical properties of the intracellular space by the actin and microtubule based cytoskeleton, we evaluated the effect of benomyl and latrunculin A. Benomyl partially decomposes the aster-like assemblies near the periphery of the cell. Latrunculin A, an actin monomer sequesterer, impedes the turnover of actin, resulting in the partial decomposition of the actin cortex.

In a third group of experiments we analysed the motion of the colloidal force probes in terms of the local velocities in the absence and presence of external forces. We show first that the distributions of absolute velocities $P(v)$ can be represented as a log-normal distribution which exhibits long tails extending to very high velocities $(v \sim 10 \mu \mathrm{m} / \mathrm{s})$ even in the absence of external forces. Parallel microfluorescence and rheological studies of cells transfected with GFP-tubulin show that the colloidal probes are in general bound to these filaments even after application of external forces up to $1 \mathrm{nN}$. The trajectories of the bead thus reflect the motion of the microtubule. From these experiments we conclude that the log-normal distributions are mainly determined by statistical sweeping motions of the microtubules about their anchoring at the centrosome with velocities of $v \sim 0.5 \mu \mathrm{m} / \mathrm{s}$ and to the intermittent motion of the beads parallel to the long axes of the microtubules (maximum velocities of $1.5 \mu \mathrm{m} / \mathrm{s}$ ). We then provide some evidence that the long tails of $P(v)$ are due to occasional fast motions of the beads that are driven by pre-stressing of the MT by forces of several hundred picoNewtons generated by local cortical tensions.

In the last part we address the question of how the viscoplastic intracellular space can maintain mechanical stability 
under the action of local external forces $(\sim 1 \mathrm{nN})$ exceeding the buckling instability limit of the microtubules $(\sim 15 \mathrm{pN})$ by orders of magnitude. We show that local forces applied to MT-coupled beads evoke immediate and rapid deflections of the filaments and of the centrosome in the direction perpendicular to or opposite to the force direction. This motion is attributed to traction forces which arise at the two filament ends (anchored at the actin cortex and the centrosome, respectively) and which generate tensions in the filaments that balance the external load. After a response time of about $0.5 \mathrm{~s}$, the filaments tend to return close to the original position by the irregular motion of the MT ends along the actin cortex (with velocities of $\sim 0.3 \mu \mathrm{m} / \mathrm{s}$ ), suggesting that the MT aster exhibits an equilibrium organization in the absence of external forces.

We finally propose a model suggesting that the mechanical stabilization of the Dictyostelia cells is provided by active coupling of the star-like assembly of microtubules to the actin cortex. This can occur by dynactin motors or other MT-actin linkers that bind the MTs to actin cables or local assemblies of the actin gels. The MT aster can be actively pre-stressed by dynactin motors or by spontaneous growth and migration of local assemblies of actin gels. We argue that this discrete mechanical design provides the cell with a high global stability while the softness of the cytoplasm enables fast, effective intracellular transport of compartments.

\section{Materials and methods}

\subsection{Preparation of cells}

D. discoideum wild-type and mutant cells were cultivated in petri dishes using liquid AX2 nutrient medium (provided by the Max-Planck-Institut für Biochemie, Martinsried, Germany). The wild-type AX2 and the myosin null mutant HS2205 [29] were transfected to express fluorescent GFP- $\alpha$ tubulin (cell lines HG1668 and HG1671 [30,31]). For each experiment, the cells were taken from axenic culture. To starve the cells, they were extracted from their nutrient by washing and centrifuging three times with cold $17 \mathrm{mM}$ $\mathrm{K}-\mathrm{Na}$ phosphate buffer ( $\mathrm{pH}$ 6.0). The supernatant was discarded after each centrifugation step and the cells were resuspended in the phosphate buffer at $4{ }^{\circ} \mathrm{C}$ by vortexing and then deposited onto the bottom glass surface of the sample chamber. The chamber was not covered in order to provide the cells with oxygen during the time of observation, since oxygen deficiency has drastic effects on the viability of the cells. The cells settled on the glass slides within minutes and the experiments were performed not later than $2 \mathrm{~h}$ after the final washing step. Under these conditions they are in the vegetative state, exhibiting a quasi-spherical shape, and do not aggregate. The cells were incubated with latrunculin A or benomyl just before the measurement. We applied a latrunculin A concentration of $5 \mu \mathrm{M}$ and washed the cells after the observation. The incubation with benomyl occurred with a concentration of $25-50 \mu \mathrm{M}$.

\subsection{Colloidal probes}

Ferromagnetic $\mathrm{Fe}_{3} \mathrm{O}_{4}$ beads of diameter $d=1.42 \mu \mathrm{m}$ were provided by W. Möller (GSF, Munich, Germany). They were separated from their solvent by centrifuging. The pellet was then re-suspended in the $17 \mathrm{mM} \mathrm{K}-\mathrm{Na}$ phosphate buffer. The suspension was agitated in a bath sonifier for $10 \mathrm{~min}$. It was further homogenized by vortexing before mixing with the cell suspension (at a cell to bead density 10:1). Uptake of the beads by the cells occurred in a rotator within about $5 \mathrm{~min}$. Experiments were started within half an hour after this step.

\subsection{The magnetic bead microrheometer}

The experimental setup was described previously $[25,32]$. The inhomogeneous field is generated with a soft iron core exhibiting a sharp wedge-shaped edge. Magnetization occurred by a magnetic coil with 1500 turns made of a $0.7 \mathrm{~mm}$ diameter copper wire. The measuring chamber (total volume of $150 \mu \mathrm{l}$ ) was made up of a Teflon frame with an $18 \mathrm{~mm} \times 18 \mathrm{~mm}$ glass slide serving as a bottom onto which the cells are deposited. The Teflon frame is embedded in a copper sample holder placed onto the microscope stage. The edge of the soft iron core penetrates into the sample chamber through an opening in the copper and Teflon housing. The penetration depth of the blade can be varied to adjust the distance to the cell studied to a minimum value of $\sim 100 \mu \mathrm{m}$. The coil current is produced by a home-made voltage-controlled current supply that transforms the voltage signal of a FG9000 function generator (ELV Elektronik AG, Leer, Germany) or DS345 (SRS Stanford Research Systems, Sunnyvale, CA, USA) into a current signal with a maximum output of $4 \mathrm{~A}$. This experimental setup was mounted onto an AXIOVERT 200 microscope (Zeiss, Oberkochen, Germany). For imaging, a Zeiss Plan-Neofluar $(100 \times \mathrm{PH} 2)$ microscope objective was used. The microscope image was recorded by a charge-coupled device camera (C 4880-80 Hamamatsu, Herrsching, Germany) and stored on a personal computer. The position of the magnetic particle was tracked by the home-made real-time image processing software OPEN BOX [33]. The time resolution was $20 \mathrm{~ms}$.

\subsection{Determination of velocity}

The local velocity of the colloidal probes in the image plane was determined by numerical differentiation according to a previously developed procedure $[7,29]$ :

$v_{\mathrm{L}}\left(x_{i}, y_{i}, t_{i}\right)=\sqrt{\left(\frac{x_{i}+1-x_{i}-1}{t_{i}+1-t_{i}-1}\right)^{2}+\left(\frac{y_{i}+1-y_{i}-1}{t_{i}+1-t_{i}-1}\right)^{2}}$

It is a coarse-grained velocity obtained by averaging the local displacements over $0.03 \mathrm{~s}$. 


\section{Experimental results and interpretations}

\subsection{Random and intermittent directed motion of magnetosomes}

To evaluate the effect of external forces on the cell cytoplasm, we analyzed 1400 responses for wild-type cells, 600 for benomyl treated cells and 480 pulses for myosin II null mutants. Fig. 1a shows a trajectory of a $1.42 \mu \mathrm{m}$ diameter magnetosome in a wild-type $D$. discoideum cell observed for $10 \mathrm{~min}$. The rainbow-like color code indicates the time course of the motion starting with red at $t_{0}=0$. The starting point is marked by the initial position of the bead (dark disk at the top). During the trajectory, force pulses of $17 \mathrm{~s}$ duration with force amplitudes ranging from 110 to $700 \mathrm{pN}$ were applied, which were always directed towards the left. The image of the cell is shown at $t=0 \mathrm{~s}$. The red, yellow, green, light blue, dark blue and purple curves (rainbow coded) in Fig. $1^{1}$ indicate the outline of the cell at $t_{\mathrm{r}}=1 \min , t_{\mathrm{y}}=2 \min 40 \mathrm{~s}, t_{\mathrm{g}}=4 \min 5 \mathrm{~s}, t_{\mathrm{lb}}=5 \min 35 \mathrm{~s}$, $t_{\mathrm{db}}=7 \min 45 \mathrm{~s}$ and $t_{\mathrm{p}}=10 \min 35 \mathrm{~s}$, respectively. The cell generates pseudopods and rotates during the observation time but it does not move significantly in a given direction. A more detailed inspection of the trajectories and global motions of the cells did not show any correlation between these two sets of motions. It should also be noted that the velocities of the advancing protrusions or rotations are on the order of $0.05 \mu \mathrm{m} / \mathrm{s}$ and are thus slow compared to the internal motions. Fig. 1a clearly shows that the trajectory of the colloidal probe is composed of local irregular walks and directed motions and this behaviour also holds if force pulses of up to $1 \mathrm{nN}$ are applied.

Closer inspection of 680 force induced trajectories shows two remarkable observations. The first is that in most cases (about $80 \%$ ) the force pulses trigger immediate fast deflections of the bead in directions opposite or perpendicular to the external force. The speed of these motions can reach $5-10 \mu \mathrm{m} / \mathrm{s}$. Below, we will provide evidence that these fast deflections are due to strong pre-stressing of the MTs (see Fig. 1c for a schematic view of the processes). The second observation is shown in Fig. 1b. A section of the trajectory of Fig. 1a is shown where several pulses P1-P4 induce a deflection of the bead nearly perpendicular to the force direction (to the left in the negative $x$-direction). The scenario starts at the top of this magnified view with the bead entering from above (light green trajectory). The bead moves first in a diffusive way towards the lower left corner of the cell and returns to the position P1. Here a pulse of $450 \mathrm{pN}$ is applied which induces an immediate and fast deflection nearly perpendicular to the force direction. The motion slows down rapidly in $\sim 1 / 2 \mathrm{~s}$ (Fig. 2). After switching off the force the bead returns to position $\mathrm{P} 2$ by irregular walks. Now a second pulse of $500 \mathrm{pN}$ is applied resulting in

\footnotetext{
${ }^{1}$ For interpretation of colour in this figure, the reader is referred to the web version of this article.
}

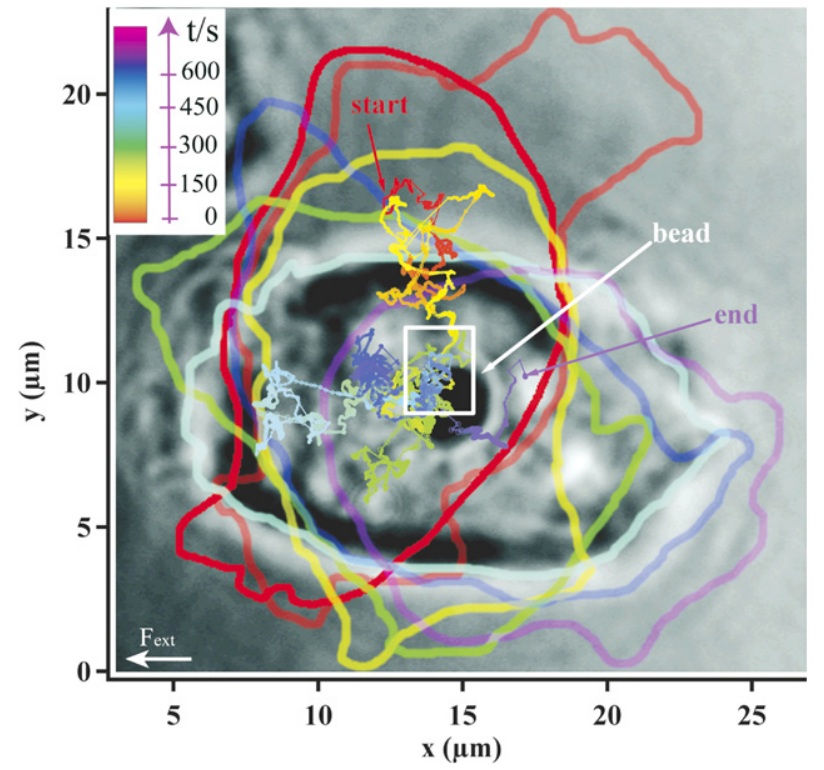

Fig. 1a. Trajectory of magnetic bead observed for about $600 \mathrm{~s}$. The movie's time flow is illustrated by a rainbow color code starting with red and ending with blue/purple. The relation between the time and the color is given in the inset. The start position of the bead is further marked by an arrow and ending in blue. The cell's contour at six distinct times is marked in red, yellow, green, light blue, dark blue and purple at the corresponding times $t_{\mathrm{r}}=1 \mathrm{~min}, t_{\mathrm{y}}=2 \min 40 \mathrm{~s}, t_{\mathrm{g}}=4 \min 5 \mathrm{~s}, t_{\mathrm{lb}}=5 \min 35 \mathrm{~s}$, $t_{\mathrm{db}}=7 \mathrm{~min} 45 \mathrm{~s}$ and $t_{\mathrm{p}}=10 \min 35 \mathrm{~s}$, respectively. The white square indicates the region shown at higher resolution in (b).

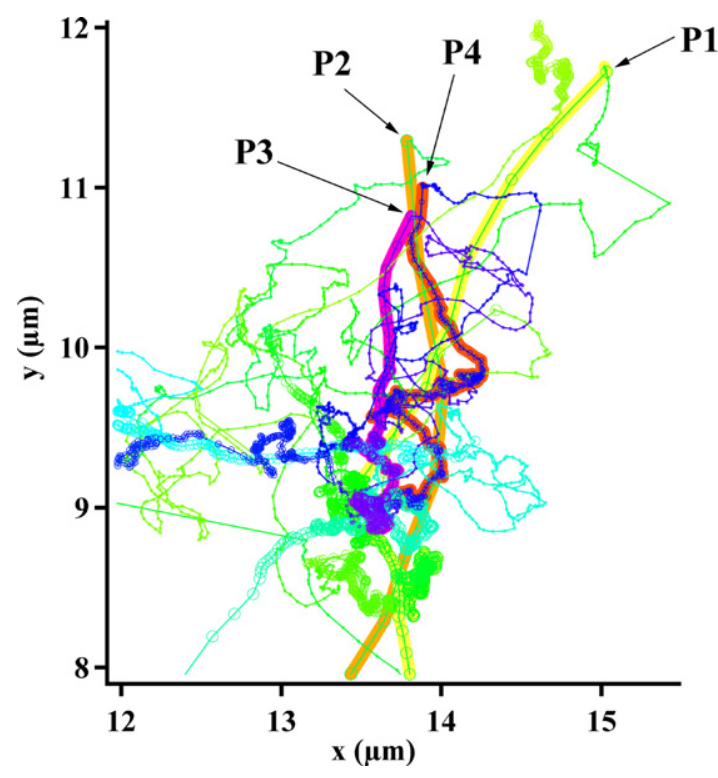

Fig. 1b. Section of bead trajectory of (a) showing situation where the bead moves predominantly perpendicular to the applied external force direction during external force pulses. The force amplitudes for pulses $\mathrm{P} 1-\mathrm{P} 4$ are $f_{1}=450 \mathrm{pN}$ (yellow line), $f_{2}=500 \mathrm{pN}$ (orange line), $f_{3}=110 \mathrm{pN}$ (red line), and $f_{4}=290 \mathrm{pN}$ (purple line), respectively. Note that due to the slow (diffusive) reversibility of the enforced deflections the bead can revisit the same region within the cell several times. Below we provide evidence that the perpendicular motion of the beads is due to angular deflections of microtubules. 


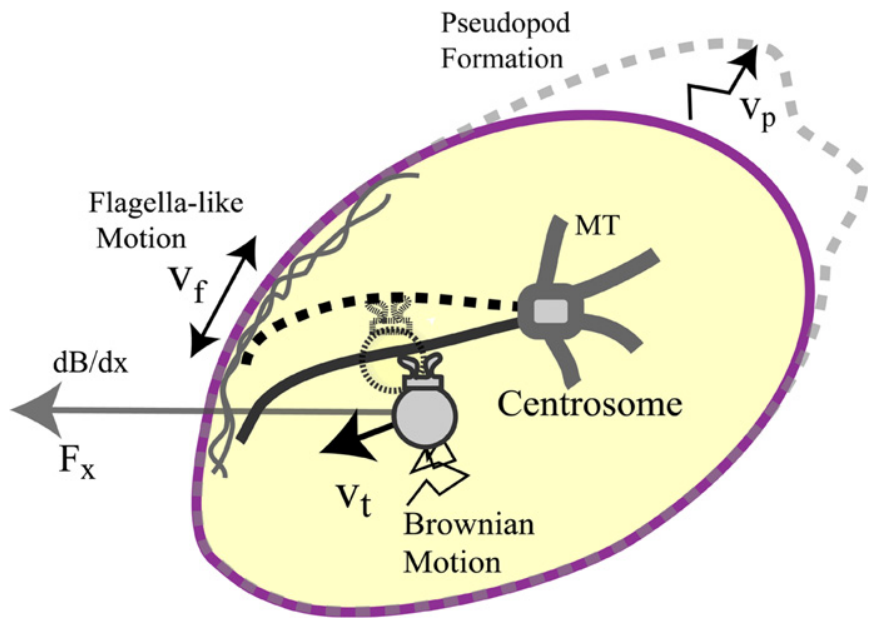

Fig. 1c. Schematic view of the relevant compartments and processes in the cell, which has internalized a magnetosome. A magnetic field gradient $\mathrm{d} B / \mathrm{d} x$ exerts a force $F_{x}$ on the magnetic bead always in the $-x$-direction. The bead moves along microtubules (MTs) with a velocity $v_{t}$. The MTs perform flagella-like motions with a velocity of $v_{f}$. $V_{\mathrm{p}}$ is the velocity of the advancement of pseudopods when the cell starts moving in a new direction. It is negligible compared to the fast bead movement in the cell.
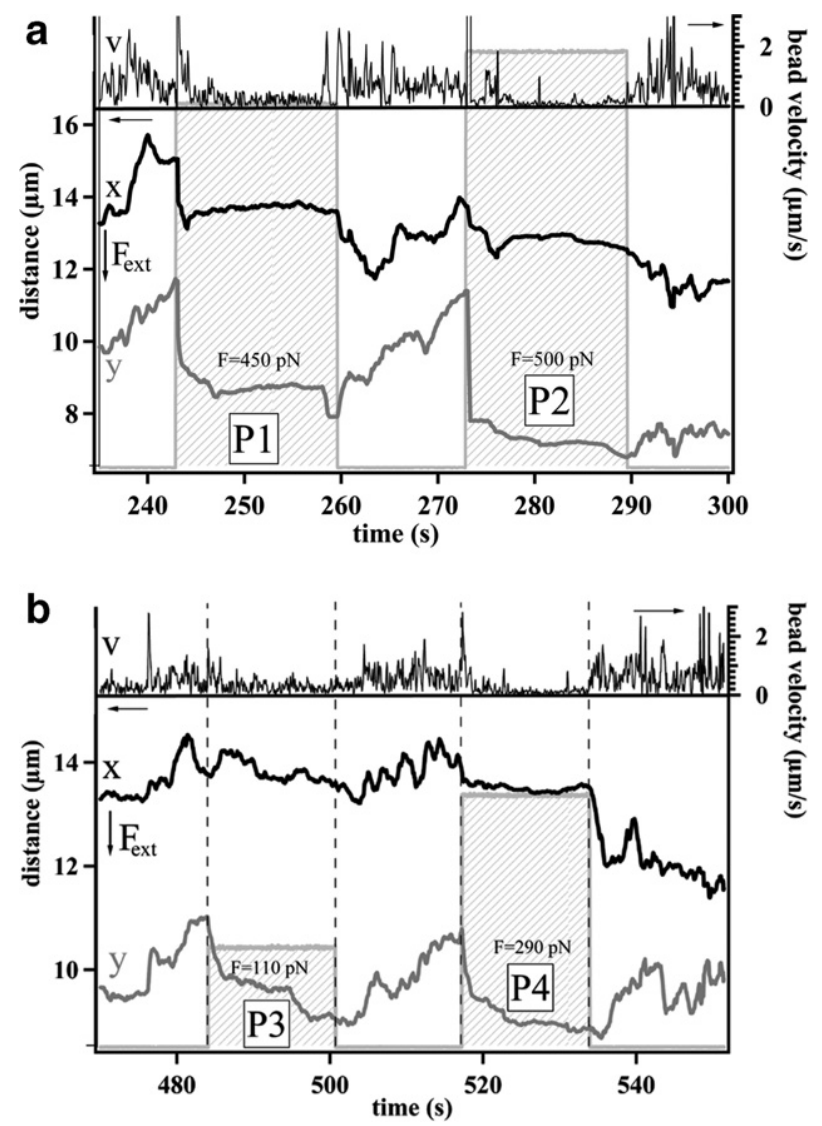

Fig. 2. Time dependence of the bead displacement in $x$-direction (thick black line) and $y$-direction (thick grey line) and of the absolute velocity (black curve on top). The velocity scale is given at the right ordinate. Note that velocities $>3 \mu \mathrm{m}$ are cut-off. The height of the grey bars reflects the force amplitudes for pulses P1-P4 $\left(f_{1}=450 \mathrm{pN}, f_{2}=500 \mathrm{pN}, f_{3}=110 \mathrm{pN}\right.$, and $f_{4}=290 \mathrm{pN}$, respectively). (a) Experimental data is shown for pulse 1 and pulse 2. (b) Experimental data is shown for pulse 3 and pulse 4. the same behaviour as before. The recurrent behaviour is observed again twice and pulses $\mathrm{P} 3(110 \mathrm{pN})$ and $\mathrm{P} 4$ $(290 \mathrm{pN})$ are applied. After the fourth pulse the bead escapes to the lower left corner without returning again. The absolute velocities and the deflections of the bead in the $x$ - and $y$-direction are shown in Fig. 2. The time dependence of the velocity $v$ clearly shows the rapid slowing down of the motion in the presence of forces above a threshold of $100 \mathrm{pN}$. At smaller forces the velocity of the bead deflection is not reduced remarkably during the application of the force.

The main observations can be summarized as follows:

Although the beads traverse about the same location in the cell several times, the velocities of deflection are very different. In the case of pulse P1 and P2 (with amplitudes of $450 \mathrm{pN}$ and $500 \mathrm{pN}$ ), the initial velocities immediately after switching on the force pulse are very high (about $v_{0} \sim 10 \mu \mathrm{m} / \mathrm{s}$ ). After a response time of about $0.5 \mathrm{~s}$ the motion is slowing down drastically. For the pulse P4 $\left(290 \mathrm{pN}\right.$ ) the initial velocity is $v_{0} \sim 1 \mu \mathrm{m} / \mathrm{s}$ and the slowing down is much less pronounced. For P3 $(110 \mathrm{pN})$ the initial velocity $(\sim 1 \mu \mathrm{m} / \mathrm{s})$ is nearly equal to the drift velocities of the bead during its return into the initial position and no remarkable slowing down is observed. The slowing down of the velocity in the presence of forces exceeding $100 \mathrm{pN}$ and the absence of this effect below this threshold force has been observed in numerous cases and appears to be a universal feature of wild-type cells. It should be noted that the return of the beads to the original position occurs only in about $50 \%$ of the 600 cases studied. It is not due to true elastic behaviour of the cytoplasm since the bead moves back in a quasi-random fashion. Below (Fig. 5) we provide evidence that the high initial velocity for P1 and P2 agrees with the initial escape velocity of the microtubules in the direction perpendicular to the applied force. The slow reversibility of the bead suggests that the star-like assembly of the filaments exhibits a stable (ground state) configuration to which the cell returns slowly after mechanical deformations.

\subsection{Velocity distributions}

The magnetic probes engulfed by the cells are wrapped by plasma membrane during the phagocytosis and are thus expected to be processed by the cell similar to intracellular compartments. Since the beads perform actively driven local irregular walks and directed motions over distances on the order of micrometers we think it is more appropriate to characterize their motion in terms of local velocity distributions instead of mean square displacement-versus- time plots [24]. Velocity distributions of nine wild-type cells with a total observation time of about $1.5 \mathrm{~h}$, six myosin II null mutants (observation time about $1 \mathrm{~h}$ ), four latrunkulin treated cells (observation time about $45 \mathrm{~min}$ ) and four benomyl treated cells (observation time about $45 \mathrm{~min}$ ) were investigated in this work. 10 min traces were analysed at time intervals of $0.06 \mathrm{~s}$ corresponding to 10,000 data point 


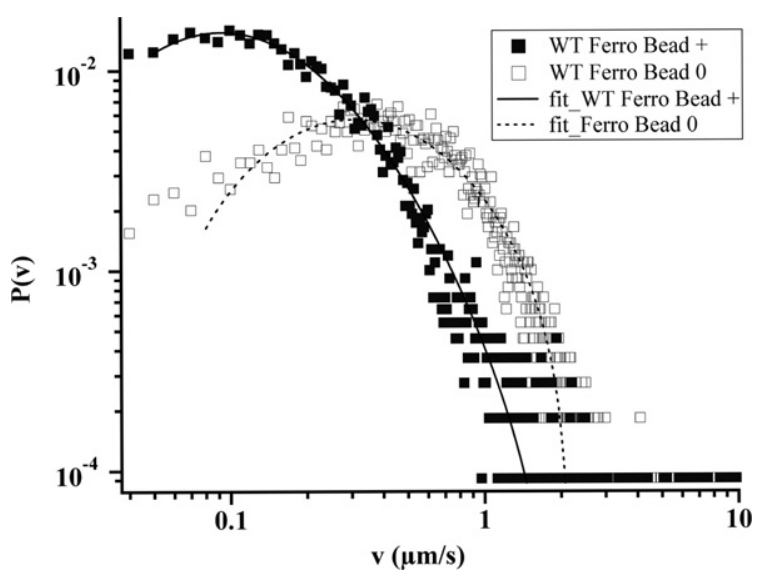

Fig. 3a. Velocity distributions of $1.42 \mu \mathrm{m}$ diameter magnetic beads in AX 2 wild-type cells in the absence ( $\square$ ) and the presence ( $\square$ ) of external force pulses are shown. The drawn curves are optimal fits of the data by lognormal distributions.

per cell. The distributions are normalized with respect to the total integral over $P(v)$. The distributions of different cells within the same cell type agreed rather well and we therefore present below the data obtained for a single cell. In Fig. 3a the velocity distributions of the magnetic bead in wild-type cells are shown for two situations: first, in the absence of external forces (open square symbols) and second, during force pulses (closed square symbols). Very broad distributions extending over three orders of magnitude in time are observed.

We attempted to characterize the velocity distributions $P(v)$ in terms of established distribution functions which are known to exhibit finite averages. We found that the measured velocity distributions between 0.02 and $2 \mu \mathrm{m} / \mathrm{s}$ can be best fitted by log-normal distributions according to

$P(v)=\frac{1}{v \sqrt{2 \pi \sigma^{2}}} \exp \left\{\frac{-(\log v)^{2}}{2 \sigma^{2}}\right\}$

See appendix for the comparison with other distribution functions. Log-normal distributions are often observed in complex systems (with fractal dimensionality) which show also 1 /frequency noise. They are typically observed if motions (such as electrons in semiconductors) are energy activated and if the relaxation times of the excitation processes driving the motion do not depend on the scale of the processes such as the length of the random walks [35]. We pointed out in our earlier studies of agar immobilized cells that the force-induced bead motion can also be described in terms of enforced motion of particles in quasi-periodic potentials [36].

The drawn curves are fits of log-normal distributions (Eq. (2)) to the measured velocity distributions. Velocities $<0.1 \mu \mathrm{m}$ could not be measured accurately enough and these data are therefore excluded from the fit. The total velocity distribution exhibits, however, long tails which can extend up to $10 \mu \mathrm{m} / \mathrm{s}$, also excluded from our fits. A surprising result is that the maximum of the distribution function $\left(P(v)_{\max }\right.$ at $\left.v \sim 0.5 \mu \mathrm{m} / \mathrm{s}\right)$ is shifted to lower values

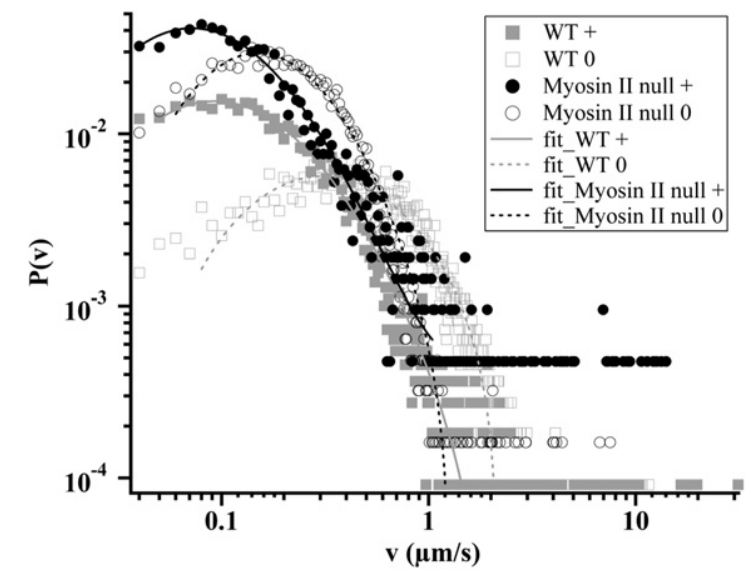

Fig. 3b. Velocity distributions of magnetic beads in myosin null mutants measured in the presence (closed black circles) and in the absence of external force pulses (open black circles). For comparison the distributions of the wild-type cell is also plotted (in grey). The open squares correspond again to the absence and the closed to the presence of forces. The drawn curves are optimal fits of the data by log-normal distributions.

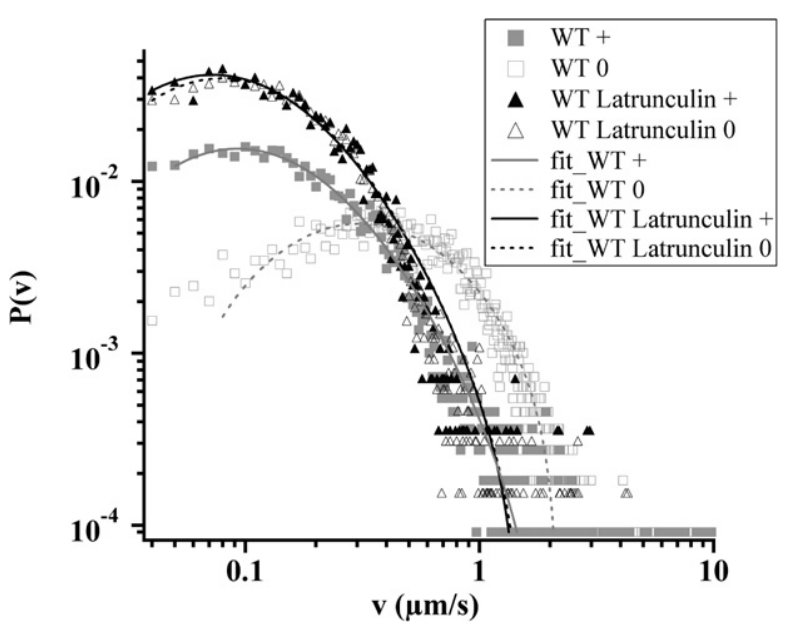

Fig. 3c. Velocity distributions of magnetic bead in latrunculin A treated wild-type cells in absence (open black triangles) and the presence of force pulses (closed black triangles). For comparison the velocity distributions of the untreated wild-type cells are shown in grey (according to (B)). The drawn curves are again optimal fits to $\log$-normal distributions.

of $v$ (to $P(v)_{\max }$ at $\sim 0.1 \mu \mathrm{m} / \mathrm{s}$ ) in the presence of external forces. This reflects the slowing down of the fast bead motion in the presence of external forces as was directly shown in Figs. 1b and 2. The effect does not depend on the relative orientation of the bead motion with respect to the external force direction and it is nearly absent below a threshold force of $100 \mathrm{nN}$.

In Fig. 3b we compare the velocity distributions of magnetic probes in myosin II mutants and wild-type cells, which were again measured in the absence and presence of external forces. It is seen that the distribution for myosin II null mutants is slightly shifted to lower velocities $\left(P(v)_{\max }\right.$ at $\left.v \sim 0.2 \mu \mathrm{m} / \mathrm{s}\right)$ with respect to wild-type cells. As in the case of wild-type cells, the maximum of $P(v)$ is again shifted to lower values under the action of external 
forces. Inspection of 480 bead responses shows that the direction of motion is still mostly directed opposite to the external force direction. This shows that knock out of myosin II does not abolish the pre-stressing of the MT and the capacity of the cell to respond actively to strong external forces. The shift of the maximum of $P(v)$ to lower velocities is explained in terms of an increase of the viscosity of the cytoplasm by knock out of myosin II. This increase of the viscosity of myosin II null mutants was observed in earlier studies $[29,34]$.

Different behaviour is found for latrunculin A treated cells. Compared to wild-type cells the maximum of $P(v)$ is shifted to lower values by a factor of five with $P(v)_{\max }$ located at $v \sim 0.1 \mu \mathrm{m} / \mathrm{s}$. Most importantly, the external forces do not induce a shift of the maximum of $P(v)$ to lower velocities demonstrating that the slowing down by external forces is abolished. This finding has been verified by direct observation of the time dependence of the velocities before and during the force pulses similar to Fig. 2. Finally, the bead is mostly deflected in the direction of the external force, indicating that the cells can no longer counteract external forces in an active way. In many cases the bead does not move at all, even after application of $700 \mathrm{nN}$ pulses, which is most likely due to the capture of the beads by microtubules near the centrosome. As will be discussed below, this is essential to prevent damage of the biochemically stressed cells by strong external forces.

The velocity distribution of benomyl treated wild-type cells exhibits similar behaviour. In these cells only short, truncated MTs are observable around the centrosome. The velocity distribution is again shifted to lower values of $v\left(P(v)_{\max }\right.$ is located at $\left.v \sim 0.1 \mu \mathrm{m} / \mathrm{s}\right)$ and external forces do not cause slowing down of the bead. Closer inspection of the trajectories of several cells shows that the beads are located predominantly close to the nucleus and are often strongly trapped near the centrosome.

\subsection{Measurement of local drag coefficients and forces from recurrent paths}

Friction coefficients could be measured in specific cases where the beads were deflected in a passive way in the force direction. In such cases the trajectories consisted of nearly straight paths with velocities altering between 0.2 and $1.0 \mu \mathrm{m} / \mathrm{s}$ (for a force of $200 \mathrm{pN}$ ) corresponding to friction coefficients between $2 \times 10^{-4}$ and $2 \times 10^{-3} \mathrm{~N} \mathrm{~s} \mathrm{~m}^{-1}$. This data corresponds well with the range of values $\zeta=2 \times$ $10^{-4}-1 \times 10^{-3} \mathrm{~N} \mathrm{~s} \mathrm{~m}^{-1}$ found for cells immobilized by agar overlays which respond to external force pulses mostly in a passive way [29]. In this study we measured active intracellular forces directly by comparing the velocities of the beads immediately before and after application of the external load and found active forces on the order of $f_{\text {act }}=100 \mathrm{pN}[36]$.

A second approach to obtain quantitative information on the time dependent drag coefficient $\zeta(t)$ of the cytoplasm is to analyze the force pulse induced deflection of the bead

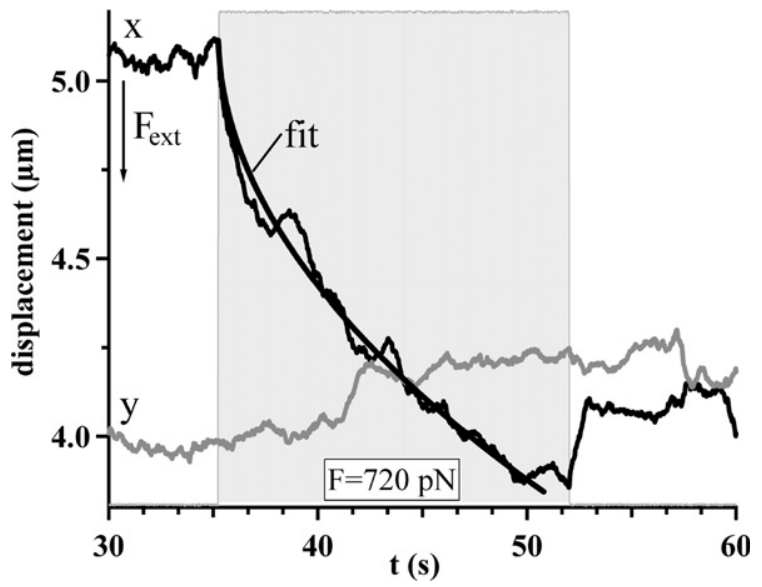

Fig. 4. Example of passive deflection of magnetic bead in force direction in latrunculin A treated wild-type cell evoked by a force pulse of $F_{\text {ext }}=720 \mathrm{pN}$. The drawn line is fitted to the data by assuming that the friction coefficient exhibits a square root law $\zeta(t) \sim 0.00015 t^{1 / 2}$.

in latrunculin A treated cells. They behave as passive viscoplastic bodies and the bead is mostly deflected in the direction of the force. In the example presented in Fig. 4 we show that the time dependence of the drag coefficient can be represented by a power law $\zeta(t)=0.0015 t^{\text {a }}$ with $a \sim$ $1 / 2$. This empirical law has been observed about 20 times for situations when the beads are not captured at the centrosome. It predicts that $\zeta$ increases from $\zeta=5 \times$ $10^{-4} \mathrm{~N} \mathrm{~s} \mathrm{~m}^{-1}$ for $t \sim 0.1 \mathrm{~s}$ (roughly the time resolution of the velocity measurement) to $\zeta \sim 5 \times 10^{-3} \mathrm{~N} \mathrm{~s} \mathrm{~m}^{-1}$ for $t \sim 10 \mathrm{~s}$ (roughly the length of the force pulses). These values are larger by about a factor 3 than the above, directly measured data, which may be explained by the closer proximity of the beads to the nuclear region. A possible explanation for this empirical power law is the accumulation of intracellular components in front of the rapidly moving bead since the intracellular compartments (such as vesicles and mitochondria) cannot escape rapidly enough by diffusion. This gives rise to a local osmotic pressure counteracting the driving force. Such behaviour has indeed been observed for entangled actin networks and it has been shown theoretically that this effect leads to a square root law of the bead motion [37]. The active forces driving the fast initial motions (such as the fast deflections shown in Fig. 1b) can now be estimated by assuming that the friction is determined by the short time value of the drag coefficient: $\zeta \sim 2 \times 10^{-4} \mathrm{~N} \mathrm{~s} / \mathrm{m}$. The fast motion of the bead along the MT (with $v \sim 1.5 \mu \mathrm{m} / \mathrm{s}$ ) would require forces of $\sim 200 \mathrm{pN}$ while $2 \mathrm{nN}$ are needed for the very fast angular deflections of the filaments (with $v \sim 10 \mu \mathrm{m} / \mathrm{s}$ ).

\subsection{Visualization of enforced motion of the microtubule}

To clarify the interaction of the beads with the microtubules during their motion, we studied cells with GFP-labelled $\alpha$-tubulin. We simultaneously observed the trajectory of the bead motion and the conformational 


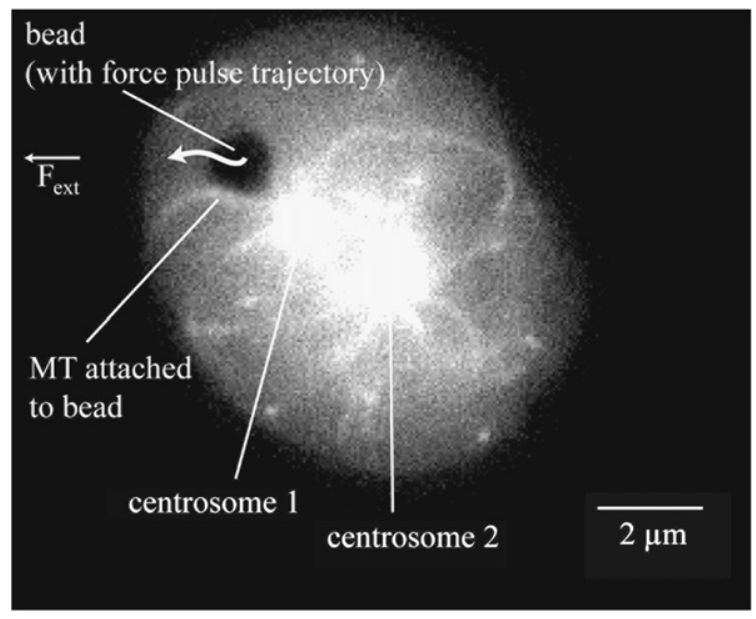

Fig. 5a. Fluorescence image of $D$. discoideum wild-type cell with two centrosomes and GFP labelled tubulin $\alpha$, at $1 \mathrm{~s}$ before an external force pulse is switched on, directed to the left ( $-x$-direction). The bead and its trajectory as well as the MT, to which it is attached, are marked. The bead trajectory contour indicates the bead trajectory during the external force pulse of $F_{\text {ext }}=40 \mathrm{pN}$. The bead follows in the direction of the applied force.

change of the MTs before and after the application of force pulses. The observation of several cells showed that the beads remain bound to microtubules most of the time even after application of pulses of several hundred picoNewtons. Several scenarios were observed. Two examples are shown in Figs. 5 and 6, where contours of the filament before and after the force pulse are shown together with the trajectories of the bead centres and of the centrosomes. For each case the MTs were imaged in time intervals of $50 \mathrm{~ms}$. This allowed us to analyze the time evolution of the conforma-

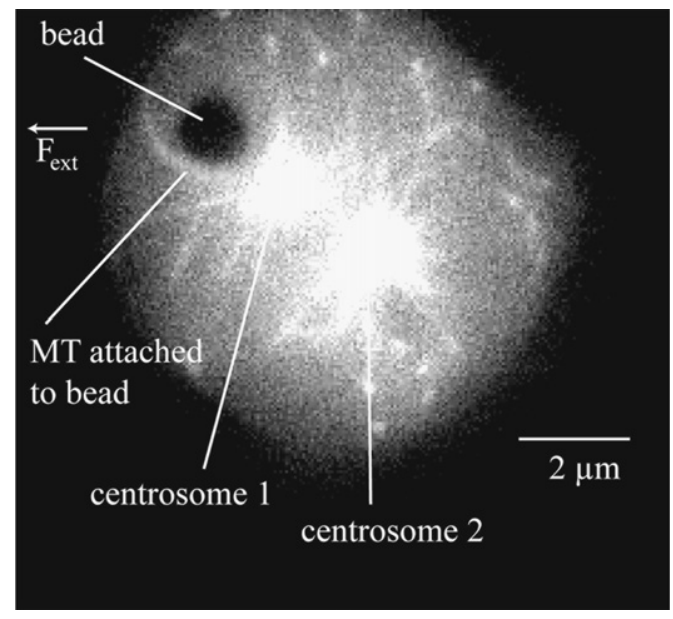

Fig. 5c. Fluorescence image of the cell during the applied external force pulse with $F_{x}=40 \mathrm{pN}$. This is a later snapshot of the movie (first image shown in (A)), from which the conformations and trajectories in (B) are extracted.

tional changes and motion of the MT in small steps and to determine the contours of the filaments in a reliable way although they were occasionally transiently blurred due to the filament motion in the vertical direction. To simplify the presentation, Figs. $5 \mathrm{~b}$ and 6 show only every sixth MT conformation observed in the movie. The bead and centrosome trajectories reflect all positions measured.

Closer inspection of the movie from which Fig. 5a and 5c are extracted shows that in the force-free state the MT filament initially rotates clockwise in a random way about the centrosome, with the end coupled to the actin cortex moving with a velocity of $v \sim 0.5 \mu \mathrm{m} / \mathrm{s}$. After application of a pulse of $f_{0} \sim 40 \mathrm{pN}$, the centrosome and the filament move per-

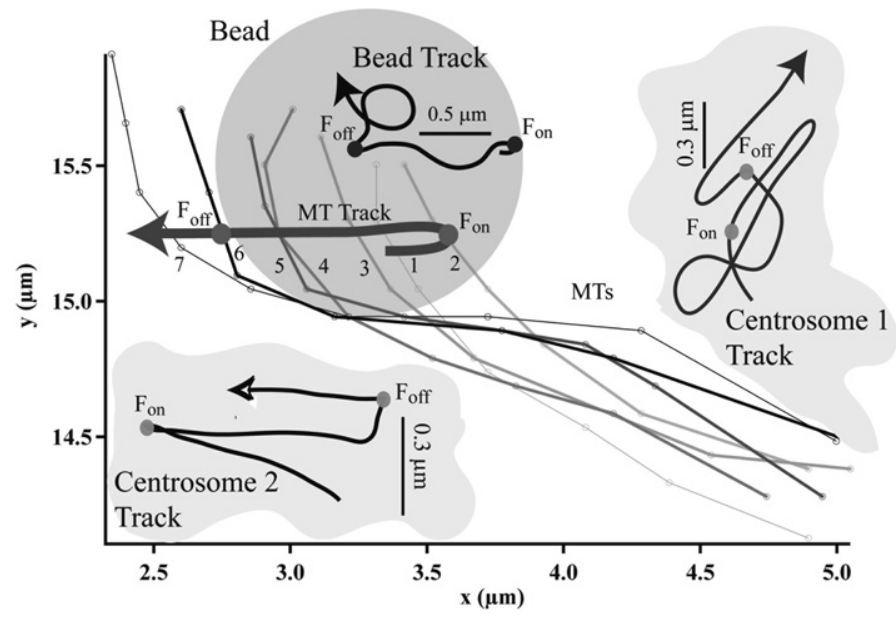

Fig. 5b. Motion of microtubule and attached bead in cell with two centrosomes, induced by a force pulse of $F_{\text {ext }}=40 \mathrm{pN}$. The thin grey line (\#1) shows a snapshot of the filament contour immediately $(56 \mathrm{~ms}$ ) before application of the force and the thick lines (numbered 2-5) during application of the force. Contour \#6 indicates the MT position after the force pulse is switched off. The thick dark grey curve with the arrow indicates the motion of the MT and is drawn to guide the eye. The time interval between contours is $0.5 \mathrm{~s}$. The inset at the right side shows the trajectory of the centrosome \#1 closer to the bead (Fig. 6A) with the dots indicating the switching on and off of the external force. The maximum initial velocity of the centrosome is $v \sim 3 \mu \mathrm{m} / \mathrm{s}$. The small inset at the top shows the corresponding trajectory of the bead centre (maximum initial velocity $\sim 1 \mu \mathrm{m} / \mathrm{s}$ ). Note that the bead follows the force direction and that the filament is elongated during the application of the force pulse with a velocity of $\sim 1 \mu \mathrm{m} / \mathrm{s}$. The inset on the bottom shows the trajectory of the remote centrosome (centrosome 2, (A)), moving in the opposite direction $(+x)$ while the external force pulse is applied in the $-x$-direction. 


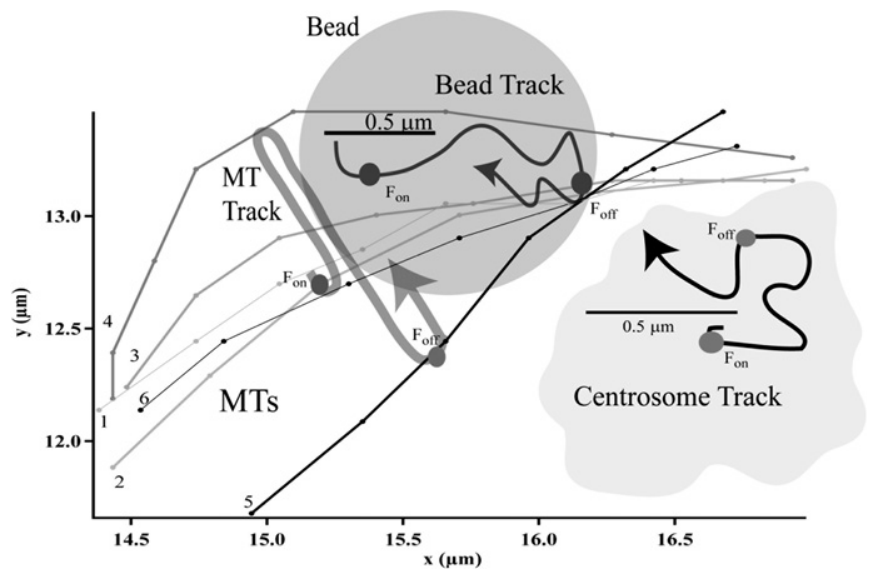

Fig. 6. Motion of microtubule and attached bead induced by a strong force pulse of $F_{\mathrm{ext}}=530 \mathrm{pN}$ in a GFP-tubulin- $\alpha$ markered Dictyostelium discoideum cell. The thin grey line (\#1) shows a snapshot of the filament contour before, the thick contours (numbered 2-5) during and the thin line \#6 after the application of the force pulse. The time intervals between snapshots were several seconds, with the exception of that between \#1 and \#2 which is $56 \mathrm{~ms}$. The thick grey line with the arrow indicates the deflection of the MT. The curve at the right (bottom) shows the trajectory of the centrosome, with the dots indicating the positions where the force is switched on and off, respectively. The initial velocity of the centrosome is $v \sim 3.5 \mu \mathrm{m} / \mathrm{s}$. The bead trajectory is given in the inset at the top of the figure, indicating that the bead moves in the opposite $(+x)$ direction with respect to the external force pulse (in the $-x$-direction). The velocity of the bead immediately after application of the force pulse is $v=4 \mu \mathrm{m} / \mathrm{s}$.

pendicular to the direction of the force pulse. The initial velocity of the centrosome after application of the force is $\sim 3 \mu \mathrm{m} / \mathrm{s}$ and that of the filament $\sim 4 \mu \mathrm{m} / \mathrm{s}$. The bead moves in the direction of the force with an initial velocity of $1 \mu \mathrm{m} / \mathrm{s}$. The filament thus responds to the force by escaping perpendicular to the force direction. This behaviour suggests that the local force generated on the filament is very rapidly compensated by traction forces acting at both ends in a direction nearly perpendicular to or opposite to the applied force (Fig. 6). After the initial phase, the filament swings back again and moves in the force direction with velocities close to those characteristic for the irregular sweeping motion of the MT (Fig. 5b). Interestingly, it is seen that the MT elongates by about $0.5 \mu \mathrm{m}$, pointing to a force induced dynamic growth [38,39].

In the case of Fig. 6, the peripheral end of the filament is initially bent downwards and the magnetic bead exerts a force tangential to the filament. After application of a $530 \mathrm{pN}$ external force, the centrosome moves immediately opposite to the force and the filament rotates counter clockwise with a high velocity of $3.5 \mu \mathrm{m} / \mathrm{s}$. It then moves back again (clockwise) more slowly $(v=0.6 \mu \mathrm{m} / \mathrm{s})$. The maximum bead velocity along the MT is $5 \mu \mathrm{m} / \mathrm{s}$, and the average velocity is $0.5 \mu \mathrm{m} / \mathrm{s}$. This shows again that the external force is very rapidly balanced by traction forces at both ends of the filament and that the cell then returns slowly again to some stable (ground) state. The velocity of this relaxation process is again similar to that of the quasi-random sweeping motions of the bead.

\section{General discussion}

\subsection{Attribution of velocity distribution to motional processes}

Inspection of the bead trajectories (Figs. 1 and 2) and the simultaneous analysis of the bead and MT motion (Figs. 5 and 6) strongly suggest that the velocity distribution (Fig. 3) is determined by three types of motions: The first consists of directed motions with velocities between 0.5 and $1.5 \mu \mathrm{m} / \mathrm{s}$ that contribute to the right wing of $P(v)$. It is largely determined by the bidirectional intermittent transport of beads in a direction parallel to the microtubules and consists of a stop-and-goes transport (with maximum velocities of $v \sim 1.5 \mu \mathrm{m} / \mathrm{s}$ ). The phases of straight motions extend over about $1 \mu \mathrm{m}$ (lasting about $0.5 \mathrm{~s}$ as judged from separate measurements of the velocity correlation function). These motions require active forces of $\sim 200 \mathrm{pN}$. Similar intermittent motions with equal maximum velocities $(\sim 2 \mu \mathrm{m} / \mathrm{s})$ are reported for axonal transports in neural cells $[38,40]$. We cannot exclude the possibility that tangential motion is partially due to the dynamic instability of the MT for which velocities of about $0.3 \mu \mathrm{m} / \mathrm{s}$ have been reported [38,39].

The second type of motion consists of flagella-like sweeping motions of the microtubules about the centrosome with velocities of $v \sim 0.5 \mu \mathrm{m} / \mathrm{s}$. They contribute mainly to the left wing of $P(v)$. Similar velocities for this type of motion were also found for cells immobilized by agar films (A. Reuter, unpublished data of Diploma Thesis, Technical University Munich, 2003). The driving force for this motion is estimated to be $F=100 \mathrm{pN}$.

The third type of motion is determined by spontaneous and very fast deflections of the MTs in random directions with velocities up to $10 \mu \mathrm{m} / \mathrm{s}$. This type of motion is similar to that induced by external forces above a threshold of $100 \mathrm{pN}$. In this case the motions consist of rapid deflections of the filaments predominantly perpendicular to the force direction. They occur concomitantly with the deflection of the centrosome (on which the filaments are anchored) in about the same direction (Fig. 5). The induced bead deflections shown in Fig. $1 \mathrm{~b}$ are attributed to this angular deflection of the MT since the initial velocities of the motions $\left(v_{0}=1.8 \mu \mathrm{m} / \mathrm{s}\right.$ and $3 \mu \mathrm{m} / \mathrm{s}$ for $110 \mathrm{pN}$ and $290 \mathrm{pN}$, respectively) agree reasonably well with the velocities of the force-induced displacement of the MT observed by microfluorescence (Fig. 5a). Driving forces in the $\mathrm{nN}$ range are needed to drive such fast motions which would require the cooperative action of several hundred motor proteins. This can hardly be achieved by MT associated motor proteins [42]. We conjecture that the fast motion is driven by pre-stressing of the microtubule by local tensions of the actin cortex generated by actin-MT crosstalk. Evidence for this conclusion is provided by the finding that the fast motions are abolished by latrunculin A. It appears to decompose the actin cortex preferentially at the interface between the actin and the MT based cytoskeletal fractions. This is suggested by our separate observation that after the 
application of this poison, the number of connections between the MTs and the actin cortex decrease rapidly with time. Moreover, the MTs appear to become much more flexible suggesting that they are not under tension anymore.

\subsection{On intracellular space as active viscoplastic bodies}

In previous studies of rheological properties of the cytoplasm we studied Dictyostelia cells immobilized by agar overlays [29]. We found that small forces between 20 and $50 \mathrm{pN}$ evoke in general bead deflections in the force direction following two types of response scenarios: (i) immediate diffusive motion in the direction of force interrupted by local random walks due to the transient capture of the beads, (ii) delayed responses with the random motions in the force direction starting with delays of several seconds after application of the force. The deflections were mostly irreversible. Similar to the mechanical behaviour of polymer melts in glassy states the friction coefficients of the cytoplasm (or the mobility of the beads) were strongly force dependent, which is the characteristic property of viscoplastic materials, together with the existence of threshold forces above which the material starts to flow [44]. This cytoplasmic viscoplasticity may provide an explanation for the glass-like behaviour of the viscoelastic impedance observed by micro-rheometric studies of fibroblasts with rotating magnetic beads [26] or by micropipette studies [14] leading to the conclusion that cells behave as glass-like bodies.

The present rheological experiments confirm this conclusion. They show, however, that the free cells behave very differently than agar immobilized cells. Their cytoplasmic space forms a much more active viscoplastic body which can rapidly balance very strong external forces in the nano-Newton regime within a fraction of a second $(\sim 0.2 \mathrm{~s})$. Such forces are also typical for the generation of lamellipodia [43].

\subsection{How the viscoplastic cytoplasmic space can generate and resist nano-Newton forces}

We now address the questions concerning the pre-stressing of the MTs and how the intracellular space can balance local forces in the nano-Newton range (e.g., applied locally to single microtubules). Since the cytoplasm is viscoplastic, the stability of the single filament can only be maintained by coupling of the aster-like assembly of microtubules to the composite and truly viscoelastic cell envelope. The maximum force which free microtubules of about $10 \mu \mathrm{m}$ length (of bending stiffness $4 \times 10^{-23} \mathrm{~J} \mathrm{~m}$ ) can resist without buckling is at most $15 \mathrm{pN}$. The micro-fluorescence observation of the induced motion of the MTs shows that mechanical stability is established by traction forces generated at the ends of the filament anchored at the centrosome and the actin cortex, respectively (Fig. 5). The force exerted by the centrosome can be balanced by the microtubules located at the opposite side of the force probe.
Tensions in the microtubule plus end coupled to the actin cortex can be generated by three possible mechanisms: first, by coupling of MT associated motor proteins such as dynactin [45] to the actin cortex (Fig. 7); second, by activation of actin/myosin micro-muscles located at the surface of the actin cortex; third, by spontaneous local growth of domain-like actin meshworks. Actin myosin bundles close to the inner surface of the cortex have been visualized by electron microscopy [41] while travelling waves of actin assemblies have recently been observed by micro-fluorescence at the growing tips of the pseudopods. Transmission of forces between the MTs and the actin cortex could be mediated by any complexes between actin and tubulin binding proteins or by vesicles with bound microtubule and actin associated motor proteins [46,47]. Myosin II motors play a key role for the positioning of microtubule asters during spindle assembly by moving the ends of the MT along the inner surface of the actin cortex [48]. However, since the pre-stresses are also observed in myosin II null mutants this mechanism is ruled out.

Two possible explanations for the mechanical stabilization of the cells are proposed (Fig. 7). One model assumes that dynactin complexes are bound to the actin cables and generate forces on the MT in the plus-end direction. According to Newton's law of force balance, an opposite force is exerted on the actin cable to which dynactin is coupled. Since the actin cortex is coupled to the plasma membrane by talin or myosin I [49] mediated links, this gives rise to a lateral cortical tension in the direction of the

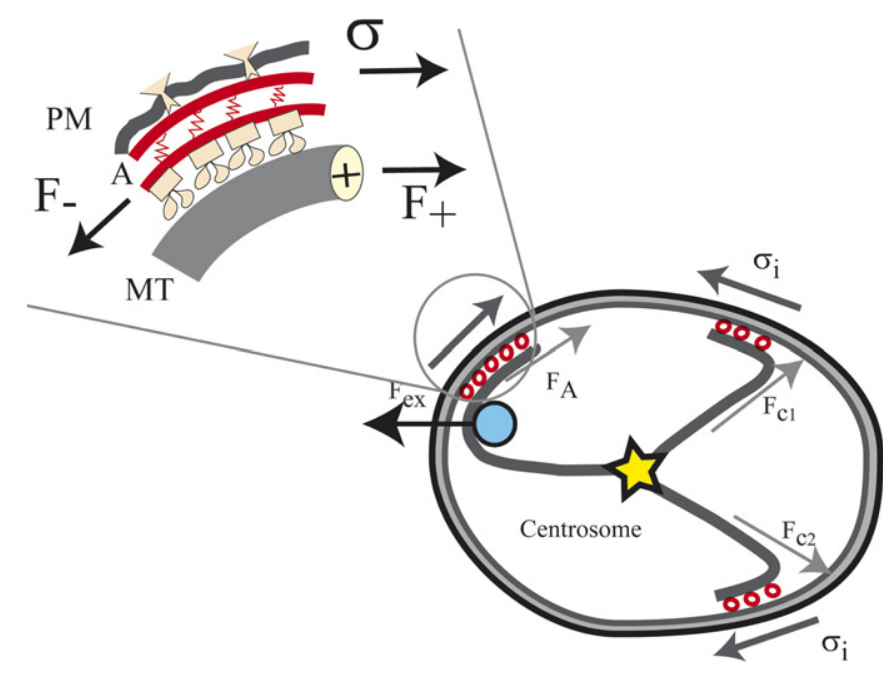

Fig. 7. Schematic view of the balance of external forces $\left(F_{\mathrm{ex}}\right)$ by generation of traction forces at the ends of the MTs anchored at the actin cortex (force $F_{A}$ ) and the centrosome (Force $F_{C}$ ), respectively. The MTs are tensed by dynactin motors generating forces in their plus-end direction. The filament tension is balanced by lateral tensions generated in the crosslinked actin cortex of the cell envelope. The force on the centrosome is stabilized by tensions generated in the MTs anchored at the opposite side of the cell. The inset shows the model of the generation of pre-stresses in the MTs by generation of cortical tensions through dynactin motors. Global stability is achieved by the balance of the tensions of the MTs and of the local cortical tensions. 
MT plus end. A force of $1 \mathrm{nN}$ would require the action of about 500 motor proteins which suggests that several MTs are involved in the generation of local cortical tensions.

A second mechanism generating pre-stresses and driving the tangential irregular walks of the actin coupled MT ends could be the spontaneous generation and decay of $\left(\sim 1 \mu \mathrm{m}^{2}\right.$ large) dynamic micro-domains of actin assemblies [50]. (These can consist of actin, crosslinked by passive linkers such as Arp2/3 [47,50] and filamin, or of actin/myosin micro-bundles.) They form mostly at the leading front of pseudopods and at the cytoplasmic side of the cell surface contacting the substrate. They migrate along the surface with typical velocities of $0.05 \mu \mathrm{m} / \mathrm{s}$ and are involved in the protrusion of pseudopods. In very recent complementary micro-fluorescence studies of cells moving over rods generated on glass surfaces, we found that these actin assemblies can move even faster $(\sim 0.5 \mu \mathrm{m} / \mathrm{s}, \mathrm{D}$. Heinrich, G. Gerisch, unpublished data) than reported in Ref. [50]. The migrating assemblies are suppressed by latrunculin but not by knock out of myosin II, in agreement with our findings. The critical buckling force of the $\sim 0.1 \mu \mathrm{m}$ long actin filaments within the meshworks (of $\sim 0.1 \mu \mathrm{m}$ mesh size) is of the order of $1 \mathrm{nN}$ [43]. Migrating actin assemblies could thus indeed generate the observed prestressing of the MT system. Finally, it should be noted that active cortical tensions can be generated by assemblies of myosin I which are coupled to actin filaments interacting with the plasma membrane through talin.

The above mechanisms can explain several of our findings. Consider first the instantaneous deflection of the MT and centrosome approximately perpendicular to the applied force $f_{\text {ex }}$ and the subsequent slow return to the original position. The force will increase the tension in the pre-stressed MT and is expected to lead to the unbinding of actin-MT connections. The local cortical tension is not balanced any more and pulls the MT opposite to the force direction until mechanical equilibrium is established again (occurring with a response time of $0.2 \mathrm{~s}$ ). This equilibration process could be mediated by tensions induced in the other MTs of the aster or by re-organization of the dynamic actin assemblies. After the local force balance is established again, the MTs return close to their original position by the irregular sweeping motions. The observation that strongly deflected microtubules often return slowly to the original position (Fig. 1) suggests that there exists some mechanical ground state providing the cell with a mechanical memory.

The above mechanisms are no longer functional after treatment of the cells with latrunculin A and benomyl, since these poisons abolish the MT-actin coupling. Our experiments suggest that the cells adapt to this situation by maintaining the beads near the nuclear region. Closer inspection of a large number of mechanical responses also suggests that the induced motion of the beads is often impeded after application of several strong force pulses. A possible explanation is that the cells adapt to strong mechanical stresses by capturing the beads again within the MT network.
In order to maintain the global stability of the cell or to avoid folding of the cell envelope, the actin cortex must form a percolated elastic network in such a way that local changes of the tangential stress can be balanced by stresses generated at other sites in the cell.

In summary, our experiments lead to the conclusion that the mechanical stability of cells exhibiting soft cytoplasms (such as Dictyostelia cells) is maintained by coupling of the microtubules to the viscoelastic actin cortex which could be mediated by binding of dynactin motors to actin cables or other linkers coupling MTs to dynamic actin assemblies [47]. Thus local external forces could be compensated by re-organization of the actin cortex until lateral cortical tensions are balanced by stresses generated within the MT aster or by both processes. Balance of cortical tensions ( $\sigma$ in Fig. 6 ) within the cell envelope alone would require that the domains of tightly crosslinked actin form a percolated network. We argue that this discrete mechanical design provides the cell with a high global stability while the softness of the cytoplasm facilitates the fast intracellular transport of compartments or the repositioning of the centrosome. The proposed model suggests some analogies between the mechanical design of Dictyostelia cells and technical tensegrity structures [51]. In the cell, the microtubules play the role of pre-stressed springs that can maintain only linear tensions while the actin cortex provides stability towards shearing and extensions. In contrast to technical tensegrity structures, cells are adaptable and can return to their original shape (mechanical ground state) by active forces. Depending on the type of experiment, the mechanical properties of the cell can be described by the continuum mode (as for the interpretation of micropipette experiments [52]) or by discrete models (such as for the evaluation of micromechanical experiments).

\section{Conclusions}

The cytoplasmic space of Dictyostelia cells behaves like a soft viscoplastic body which is mechanically stabilized by active coupling of the aster-like assembly of microtubules to the actin cortex. This could be mediated either actively by motors of the dynactin family or passively by coupling of MTs to dynamic actin assemblies. The actin cortex and the MT aster form an interconnected (percolated) mechanical structure in such a way that sudden local loads can be balanced by cortical tensions progressing within the actin cortex, by stresses generated within the MT aster or by both mechanisms. Local fluctuations of the cortical tensions induce flagella-like motions of the MTs and quasirandom motions of the centrosome, which can lead to strong pre-stressing of the MTs.

This discrete mechanical design allows cells to resist nano-Newton mechanical forces while the cytoplasm forms a soft viscous body which is essential for the rapid intracellular transport. This design of Dictyostelia cells shows some analogies to that of technical tensegrity structures, 
with the MTs playing the role of pre-stressed springs that can maintain only linear tensions while the actin cortex provides the stability against shearing.

\section{Acknowledgements}

The work was supported by the Volkswagenstiftung and the Fonds der Chemischen Industrie. We thank Prof. G. Gerisch for providing the cells and for very helpful discussions. One of the authors (D.H.) acknowledges the hospitality of the group of Prof. J. Spatz at the University of Heidelberg and the help of the Nikon Imaging Center with complementary micro-fluorescence experiments.

\section{Appendix}

We compared the fits of three different distributions to the measured velocity distribution functions presented in Fig. 3a:

Log-normal distribution: $P(v)=K_{0}+K_{1} * \exp (-(\ln (x)$ $\left.\left.K_{2}\right) / K_{3}\right)$ ).

Exponential distribution: $P(v)_{\mathrm{ex}}=K_{0}+K_{1} * \exp \left(-K_{2} * x\right)$. Power law distribution: $P(v)_{\mathrm{P}}=K_{0}+K_{1} * x^{K_{2}}$

Table 1

Fitting parameters and $\chi^{2}$ values for the optimal fits (log-normal, exponential and power law) in the restricted range of the velocities $(v=0.05-1.5 \mu \mathrm{m} / \mathrm{s})$ for wild-type cells (Fig. 3a) in the presence and absence of external forces

\begin{tabular}{lcc}
\hline & With force applied & No force applied \\
\hline Log-normal fit & & \\
$\chi^{2}$ & $4.38159 \mathrm{e}-5$ & $7.02617 \mathrm{e}-5$ \\
$K_{0}$ & $-4.40819 \mathrm{e}-5$ & -0.000782318 \\
$K_{1}$ & 0.0155455 & 0.00645721 \\
$K_{2}$ & 0.0926804 & 0.296585 \\
$K_{3}$ & 1.26819 & 1.40169 \\
Exponential fit $_{\chi^{2}}$ & & \\
$K_{0}$ & 0.000101386 & 0.000187522 \\
$K_{1}$ & $-4.64562 \mathrm{e}-5$ & -0.00523198 \\
$K_{2}$ & 0.0213083 & 0.010871 \\
Power law fit & 3.87424 & 0.345876 \\
$\chi^{2}$ & & \\
$K_{0}$ & 0.000363005 & 0.00035964 \\
$K_{1}$ & -0.0049732 & -0.0597949 \\
$K_{2}$ & 0.000017008 & 0.0617602 \\
\hline
\end{tabular}

Table 2

Fitting parameters and $\chi^{2}$ values for the optimal log-normal fit over the whole range of velocities with the exception of the long tails at $v>4 \mu \mathrm{m} / \mathrm{s}$

\begin{tabular}{lcc}
\hline & With force applied & No force applied \\
\hline Log-normal fit & & \\
$\chi^{2}$ & $7.66131 \mathrm{e}-5$ & $7.96925 \mathrm{e}-5$ \\
$K_{0}$ & -0.000100558 & -0.000267676 \\
$K_{1}$ & 0.0159749 & 0.00603335 \\
$K_{2}$ & 0.0777968 & 0.299752 \\
$K_{3}$ & 1.42855 & 1.2694 \\
\hline
\end{tabular}

The parameters are summarized in Table 1. The range of velocities over which the measured distributions (with and without applied force) could be fitted at all with the exponential and power law distributions had to be reduced both at the beginning and at the end of the velocity scale leaving a fitting range between $v=0.05$ and $1.5 \mu \mathrm{m} / \mathrm{s}$. It should be noted that the exponential and power law distributions can fit reasonably well only the decreasing branch of the velocity distributions, while the log-normal distribution can fit the ascending and decreasing branches of distributions over the whole range of velocities. In Table 2 we present the fit parameters for the log-normal distribution obtained by fitting $P(v)$ over the whole range of measured velocities.

\section{References}

[1] Boal D. Mechanics of the cell. Cambridge: Cambridge University Press; 2002.

[2] Bray D. Cell movements: from molecules to motility. New York: Garland Publishing; 2001.

[3] Sackmann E, Bruinsma R. Cell adhesion as wetting transition. Chem Phys Chem 2002;3:262-9.

[4] Grill S, Howard J, Schäffer E, Stelzer FHK, Hyman A. The distribution of active force generators controls mitotic spindle position. Science 2003;301:518-21.

[5] Hall A. Rho GTPases and the actin cytoskeleton. Science 1998;279:509-14.

[6] Alon R, Feigelson S. From rolling to arrest on blood vessels: leucocyte tap dancing on endothelial cells integrin ligands and chemokines at sub-second contacts. Immunology 2002;14:93-104.

[7] Feneberg W, Aepfelbacher M, Sackmann E. Microviscoelasticity of the apical cell surface of human umbilical vein endothelial cells (HUVEC) within confluent monolayers. Biophys J 2004;87:1338-50.

[8] Vonna L, Aepfelbacher M, Sackmann E. Local force induced protrusions of phagocytic cells. J Cell Sci 2003;116:785-90.

[9] Zheng J, Lamoureux P, Santiago V, Dennerll RE, Buxbaum RE, Heidemann SR. Tensile regulation of axonal elongation and initiation. J Neurosci 1991;11:1117-25.

[10] Yamada X, Wirtz D, Kuo SC. Mechanics of living cells measured by laser tracking microrheometry. Biophys J 2000;78:1736-47.

[11] Ingber DE, Heidemann SR, Lamoureux P, Buxbaum RE. Opposing views on tensegrity as a structural framework for understanding cell mechanics. J Appl Physiol 2000;89:1663-78.

[12] Evans EA, Yeung A. Apparent viscosity and cortical tension of blood granulocyte determined by micropipette aspiration. Biophys $\mathrm{J}$ 1989;5:151-60.

[13] Drury J, Dembo M. Mechanics of neutrophils. Synthetic modelling of three experiments. Biophys J 2001;81:3166-77.

[14] Zhelev D, Hochmuth RM. Mechanical stimulated cytoskeleton rearrangement and cortical contraction in human neutrophils. Biophys J 1995;68:2004-14.

[15] Rotsch C, Radmacher M. Drug-induced changes of cytoskeletal structure and mechanics in fibroblasts: an atomic force microscopy study. Biophys J 2000;78:520-35.

[16] Choquet D, Felsenfeld D, Sheetz M. Extracellular matrix rigidity causes strengthening of integrin-cytoskeleton linkage. Cell 1997;88:39-48.

[17] Mills JP, Qie L, Dao M, Lim CT, Suresh S. Nonlinear elastic and viscoelastic deformation of the human red blood cell with optical tweezers. MCB 2004;1:169-80.

[18] Pasternak C, Wong S, Elson EL. Mechanical function of dystrophin in muscle cells. J Cell Biol 1995;128:355-61.

[19] Sackmann E, Bausch AR, Vonna V. Physics of composite cell membrane and actin based cytoskeleton. Nato Advanced Study 
Institute. In: Houches Les, Flyvberg H, et al., editors. Physics of biomolecules and cells, Session LXXV. Berlin: Springer Verlag; 2002.

[20] Bischofs IB, Safran SA, Schwarz US. Elastic interactions of active cells with soft materials. Phys Rev E 2004;69:021911.

[21] Suresh SJ, Spatz J, Mills JP, Micoulet A, Dao M, Lim CT, et al. Connection between single cell biomechanics and human disease states: gastrointestinal cancer and malaria. Acta Biomater 2005;1:15-31.

[22] Glogauer M, Ferrier J, McCulloch CA. Magnetic fields applied to collagen-coated ferric oxide beads induce stretch-activated $\mathrm{Ca}^{2+}$ flux in fibroblasts. Am J Physiol 1995;269:C1093-104.

[23] Crocker JC, Valentine MT, Weeks ER, Gisler T, Kaplan PD, Yodh AG, et al. Two-point microrheology of inhomogeneous soft materials. Phys Rev Lett 2000;85:888-91.

[24] Caspi A, Granek R, Elbaum M. Diffusive and directed motion in cellular transport. Phys Rev E 2002;66:011916-25.

[25] Bausch AR, Moeller W, Sackmann E. Measurement of local viscoelasticity and forces in living cells by magnetic tweezers. Biophys J 1999;76:573-9.

[26] Fabry B, Maksym GN, Butler JP, Glogauer M, Navajas D, Fredberg JJ. Scaling the microrheology of living cells. Phys Rev Lett 2001;87:148102.

[27] Wilhelm C, Gazeau F, Bacri JC. Rotational magnetic endosome microrheometry: viscoelastic architecture inside living cells. Phys Rev E 2003;67:061908-1-8-2.

[28] Rowat AC, Foster LJ, Nielsen MM, Weiss1 M, Ipsen JP. Characterization of the elastic properties of the nuclear envelope. J Roy Soc Interf 2005;2:63-9.

[29] Feneberg W, Westphal M, Sackmann E. Dictyostelia cells cytoplasm as an active viscoplastic body. Eur Biophys J 2001;30:284-94.

[30] Manstein DJ, Titus MA, Lozanne AD, Spudich JA. Gene replacement in Dictyostelium: generation of myosin null mutants. EMBO J 1989;8:923-32.

[31] Neujahr R, Albrecht R, Köhler J, Matzner M, Schwartz JM, Westphal M, et al. Microtubule-mediated centrosome motility and the positioning of cleavage furrows in multinucleated myosin II-null cells. J Cell Sci 1998;111:1227-40.

[32] Ziemann J, Raedler J, Sackmann E. Local measurements of viscoelastic moduli of entangled actin networks using an oscillating magnetic bead microrheometer. Biophys J 1994;66:2210-6.

[33] Schilling JE, Sackmann E, Bausch DA. Digital image processing for biophysical applications. Rev Sci Instrum 2004;75:2822-6.

[34] Merkel R, Simson R, Simson DA, Hohenadl M, Boulbitch A, Wallraff A, et al. Micromechanic study of cell polarity and plasma membrane cell body coupling in Dictyostelium. Biophys J 2000;79:707-19.

[35] Montroll EW, Schlesinger MF. On $1 q / f$ noise and other distributions with long tails. Proc Natl Acad Sci 1982;79:3380-3.

[36] Sackmann E, Reuter A, Heinrich D. Micromechanics of cells: viscoelastic microscopy of cells. In: Gutkovski W, Kowalevski T, editors. Mechanics of the 21st century-ICTAM'04 proceedings. Dordrecht: Kluwer Academic Publishers; 2005. p. 219-24.

[37] Uhde J, Feneberg W, Ter-Oganessian N, Sackmann E, Boulbitch A. Osmotic force-controlled microrheometry of entangled actin networks. Phys Rev Lett 2005;94:198102-1-2-5.

[38] Pollard TO, Earnshaw WC. Cell biology. Philadelphia (PA): W.B. Saunders; 2002.

[39] Mandelkov EM, Mandelkov E, Milligan RA. Microtubule dynamics and microtubule caps: a time resolved cro-electron microscopy study. J Cell Biol 1991;114:977-91.

[40] Schutz GJ, Axmann M, Freudenthaler S, Schindler H, Kandror K, Roder JC, et al. Visualization of vesicle transport along and between distinct pathways in neuritis of living cells. Microsc Res Tech 2004;63(3):159-67.

[41] Ogihara S, Carboni J, Condeelis C. Electron microscopic localization of myosin II and ABP-120 in the cortical actin matrix of Dictyostelium amoebae using IgG-gold conjugates. Dev Genetics 1988;9:505-20.

[42] Jay PY, Pham PA, Wong SA, Elson EL. A mechanical function of myosin II in cell motility. J Cell Sci 1995;108:387-93.

[43] Laddam G, Vonna L, Sackmann E. Protrusion force transmission of amoeboid cells crawling on soft biological tissue. Acta Biomater 2005;1:485-91.

[44] Argon AS. Plastic deformation in metallic glasses. Acta Metall 1979;27:47-58.

[45] Koonce MJ, Koehler J, Neujahr R, Schwartz JM, Tikonenko I, Gerisch G. Dynein motor regulation stabilizes interphase microtubule arrays and determines centrosome position. EMBO J 1999;18: 6786-6792.

[46] Goode B, Drubin D, Barnes G. Functional cooperation between microtubuli and actin cytoskeleton. Curr Opin Cell Biol 2000;12:63-71.

[47] Janmey P. The cytoskeleton and cell signalling component localisation and mechanical coupling. Physiol Rev 2001;78:763-81.

[48] Rosenblatt J, Cramer LP, Baum B, McGee M. Myosin II dependent cortical movement is required for centrosome separation and positioning during mitotic spindle assembly. Cell 2004;117:361-72.

[49] Dai JH, Ping Ting-Beall, Hochmuth RM, Sheetz MP, Titus MA. Myosin I contributes to the generation of resting tension. Biophys $\mathbf{J}$ 1999;77:1168-76.

[50] Insall R, Mueller-Taubenberger A, Machesky L, Koeler J, Simeth E, Atkinson SJ, et al. Dynamics of the Dictyostelium Arp2/3 complex in endocytosis, cytokinesis and chemotaxis. Cell Motil Cytoskel 2001;50:115-28

[51] Wendling SP, Canadas P, Chabran P. Towards a generalized tensegrity model describing the mechanical behaviour of the cytoskeleton structure. Comput Meth Biomech Biomed Eng 2003;6:45-52.

[52] Hochmuth RM. Micropipette aspiration of living cells. J Biomech 2000;33:15-22. 